\title{
Localized Electronic Excitations in Phenylacetylene Dendrimers
}

\author{
Sergei Tretiak, Vladimir Chernyak, and Shaul Mukamel* \\ Department of Chemistry and Rochester Theory Center for Optical Science and Engineering, \\ University of Rochester, P.O. RC Box 270216, Rochester, New York 14627-0216
}

Received: January 9, 1998; In Final Form: March 4, 1998

\begin{abstract}
Electron-hole pairs created upon optical excitation of conjugated dendrimers (fractal antenna macromolecules) are shown to be localized within segments connected by benzene rings substituted at the meta-position. The absorption spectra of two families of dendrimers are analyzed using collective electronic normal modes representing the changes in charge and bond-order distributions induced by the optical field. The present approach may be used in the design of artificial light-harvesting antennae with controlled energy-funneling pathways.
\end{abstract}

\section{Introduction}

Dendrimeric molecules with branched treelike structures are drawing considerable attention. ${ }^{1-7}$ The dynamics of photophysical (electronic and vibrational energy transfer) as well as photochemical processes has been demonstrated to be strongly affected by geometric confinement. Theoretical interest in these "Cayley trees" (also known as Bethe lattices) arises from their peculiar dimensionality. The connectivity between different sites is one-dimensional (there is only one path to go between two points); however, the number of atoms grows exponentially with generation, as in infinite-dimensional systems. This leads to unusual transport and optical properties. Calculating the optical electronic excitations in these systems is a formidable task, and no simple method exists for analyzing the nature of these excitations and predicting their scaling with molecular size.

In this paper we apply a novel collective electronic oscillator (CEO) approach ${ }^{8,9}$ for calculating the absorption spectra of two families of dendrimers made up of a phenylacetylene repeat unit in a self-similar fashion around the core (see Figure 1). These macromolecules have been suggested as artificial photonic antennae. ${ }^{7,10-13}$ The spectral frequency profile of family A, which has the same segment (linear unit) length in the various generations, is unchanged across the series of molecules (see bottom two panels in Figure 2). Family B has a varying segment length that increases for higher generations. Here the absorption spectra displayed in the top three panels in Figure 2 show new red-shifted features as the molecular size is increased. Our analysis shows how these trends follow naturally from the localized electronic excitations in these systems. It is difficult to anticipate this localization by inspecting the molecular orbitals since the system is conjugated and the orbitals are delocalized. ${ }^{14,15}$ Nevertheless, we demonstrate that the electron-hole pairs that contribute to the elementary optical collective excitations are well-localized.

The first step in our analysis is to compute the reduced singleelectron density matrix ${ }^{16} \bar{\rho}_{m n} \equiv\left\langle g\left|c_{m}^{+} c_{n}\right| g\right\rangle$, where $c_{m}^{+}\left(c_{m}\right)$ are creation (annihilation) operators of an electron at the $m$ th atomic orbital and $|g\rangle$ is the ground-state many-electron wave function. The diagonal elements $\bar{\rho}_{n n}$ represent the electronic charge density at the $n$th orbital, whereas the off-diagonal elements, $m \neq n$, reveal the bonding structure (i.e., bond order) associated with each pair of atomic orbitals. ${ }^{16-18}$ When the molecule interacts with an external driving field, its electronic density matrix acquires a time-dependent component $\delta \rho(t)$, which can be expanded as

$$
\delta \rho(t)=\sum_{v} a_{v}(t) \xi_{v}+a_{v}^{*}(t) \xi_{v}^{+}
$$

$a_{v}(t)$ are time-dependent expansion coefficients and the electronic normal mode $\xi_{v}$ is a matrix representing the optical transition between the ground-state $|g\rangle$ and an electronically excited state $|v\rangle$. Its matrix elements are given by

$$
\left(\xi_{v}\right)_{m n}=\left\langle v\left|c_{m}^{+} c_{n}\right| g\right\rangle
$$

Normal modes, which are commonly used in the description of molecular vibrations, are collective coordinates that represent coherent motions of the various atoms. Similarly, the electronic normal modes allow us to interpret and visualize the changes induced in electronic structure upon optical excitations in terms of collective motions of the electronic density matrix.

The electronic modes represent collective motions of electrons and holes. They carry substantially less information than the many-electron eigenstates but more than required for calculating molecular polarizabilities and spectroscopic observables. The diagonal elements $\left(\xi_{v}\right)_{n n}$ represent the net charge induced on the $n$th atomic orbital by an external field, whereas $\left(\xi_{v}\right)_{m n} n \neq$ $m$ are the dynamical bond orders (or electronic coherences) representing the joint amplitude of finding an electron on orbital $m$ and a hole on orbital $n$. Even though eq 1.2 involves matrix elements of the global many-electron eigenstates $|v\rangle$ and $|g\rangle$, the CEO can be computed as eigenmodes of the linearized timedependent Hartree-Fock (TDHF) equations of motion for the time-dependent density matrix driven by the external field, totally avoiding the calculation of many-electron eigenstates. The eigenfrequencies $\Omega_{v}$ of these equations constitute the optical transition frequencies, ${ }^{8,9}$ and the absorption spectrum is given by

$$
\alpha(\omega)=\sum_{v} \frac{f_{v} \Gamma_{v}}{2 \Omega_{v}} \frac{1}{\left(\omega-\Omega_{v}\right)^{2}+\Gamma_{v}^{2}}
$$

where $f_{v}=2 \Omega_{v}\left[\operatorname{Tr}\left(\mu \xi_{v}\right)\right]^{2}$ is the oscillator strength of the $|g\rangle$ to $|v\rangle$ transition, $\Gamma_{v}$ is the linewidth, and $\mu$ is the dipole moment operator. 
A



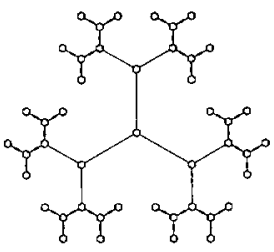

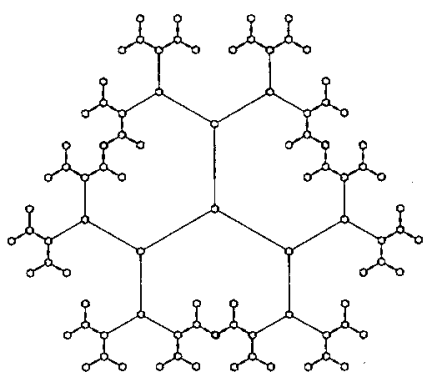

D-4 D-10 D-22

D-46

D-94

B<smiles>CC(C)C(C(C)C)C(C(C)C)C(C)(C)C</smiles>

D-25

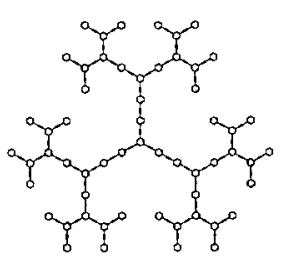

D-58

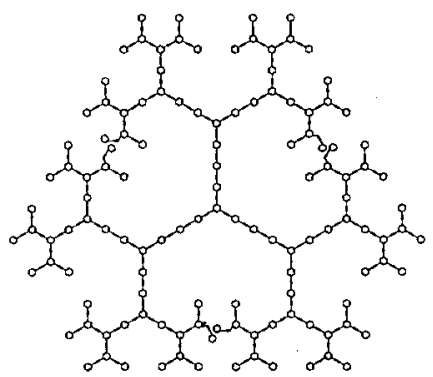

D-127
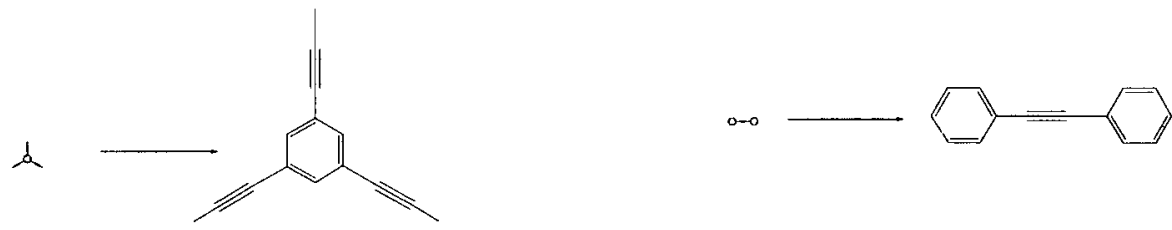

Figure 1. Structures of the two families of phenylacetylene dendrimers. ${ }^{11}$ The compact dendrimers (A) are made of the same linear building block $\mathrm{P} 1$. The extended dendrimers (B) have a varying linear unit length which decreases for higher generations.

The CEO approach provides a simple computational algorithm for optical excitations of large and complex molecules with hundreds of heavy atoms. Furthermore, the resulting modes allow a direct real-space interpretation of these spectra, illustrating the roles of geometric confinement and exciton localization. In section II we analyze the absorption spectra and the electronic modes of the phenylacetylene oligomers shown in Figure 3, which are the basic building blocks of the dendrimers (Figure 1). Using these modes we then compute the absorption spectra of phenylacetylene dendrimeric macromolecules in section III. Our calculations establish the existence of localized optical excitations and show how all the observed trends with dendrimer size and geometry follow directly from this localization. Strategies for the synthesis of antennae with specified energy funneling to a desired active site follow directly from our analysis.

\section{Collective Electronic Excitations in Linear Oligomers}

The dendrimers shown in Figure 1 are made out of phenylacetylene oligomer segments connected through para- or metasubstitutions of the benzene rings, leading to linear or zigzag chains respectively. ${ }^{11-13}$ Understanding the electronic excitations of these segments should be the first step in analyzing the dendrimer spectra. In this section we examine the linear (parasubstituted) molecules denoted $\mathrm{P} n$ with $n=1,2,3,7$ repeat units (triple bonds) and the M7 molecule made of linear P1,

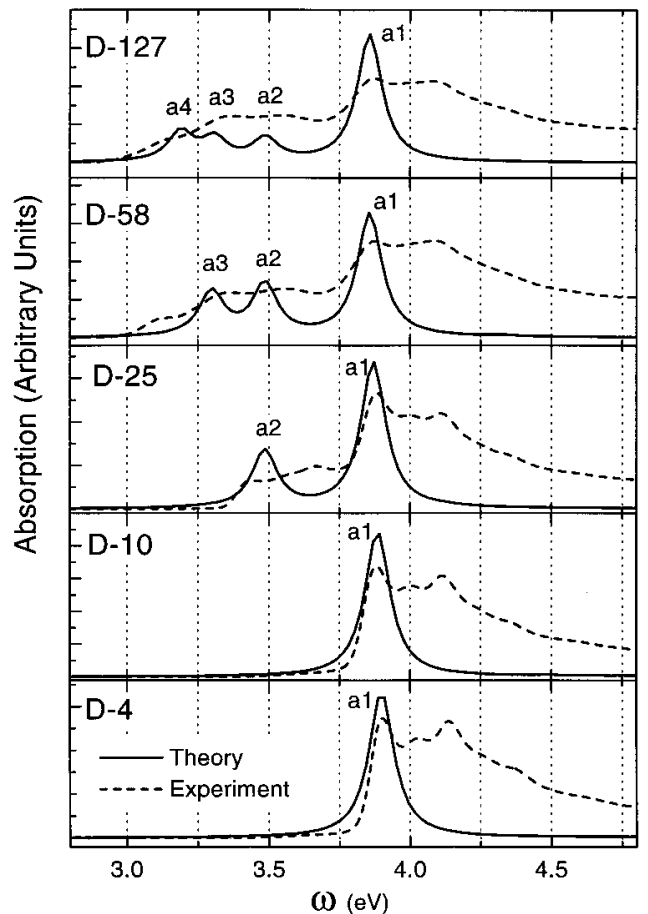

Figure 2. Calculated (solid lines) and experimental ${ }^{11}$ (dashed lines) absorption spectra of the dendrimers shown in Figure 1. Bottom two panels, family A; top three panels, family B. 




Figure 3. Structures and atom labeling of the linear para-oligomers Pn with $n=1,2,3,7$ repeat units (triple bonds) and the M7 oligomer made of the P1, P2, P3 units conjugated at the meta-position.

P2, P3 segments connected at the meta-position with overall $n$ $=7$ repeat units (see Figure 3 ). Molecules P7 and M7 are made of the same segments, have the same size, and only differ by geometry (meta- vs para-substitutions). The notable difference between their spectra turns out to be the key for our analysis.

We first calculated the Hartree-Fock ground-state density matrixes $\rho_{m n}$. Optimal ground-state geometries were obtained at the AM1 level using Gaussian-94. The ZINDO code was used next to generate the INDO/S Hamiltonian, ${ }^{19-21}$ and the CEO/DSMA procedure ${ }^{9}$ was finally applied to compute the linear absorption spectra. Satisfactory convergence of the linear absorption was achieved using 10-15 effective electronic modes.

The calculated linear absorption spectra (eq 1.3) are shown in Figure 4. The P-oligomers have two major absorption lines in the $3-6 \mathrm{eV}$ frequency range. The band-edge transition a is significantly red-shifted as the chain length is increased, whereas the second peak $b$ only shows a small red-shift. ${ }^{8}$ The $5.6 \mathrm{eV}$ mode b of M7 is similar to P7(b). However, the a transition is markedly different; the single line is now split into three lowfrequency transitions (a1, a2, and a3) at the same frequencies as the band-edge transitions in P1, P2, and P3 oligomers, which are the building linear blocks of this molecule.

To explore the nature of the electronic motions underlying the various peaks and to establish a direct real-space link between the optical response and the dynamics of charges induced by optical excitation, we examined the collective modes corresponding to these electronic excitations. Contour plots of the ground-state density matrices $\bar{\rho}_{m n}$ are shown in Figure 5. The size of the matrices is equal to the number of carbon atoms in the molecule, labeled according to Figure 3. Panel P1 $(\rho)$ displays the ground-state density matrix $\bar{\rho}$ of P1. It is dominated by the diagonal and near-diagonal elements, reflecting the bonds between nearest neighbors. The aromatic rings (corners of the matrix) and the acetylenic triple bond at the center are clearly visible. Panels P7 $(\rho)$ and M7 $(\rho)$ show the ground-state density matrices of P7 and M7. The two density matrices are very similar and may be constructed by repeating the P1 density matrix: para- and meta-conjugations make a very little difference as far as the ground state is concerned. This is, however, not the case for the electronic normal modes $\left(\xi_{v}\right)_{m n}$ responsible for optical excitations, displayed in Figure 6. The ordinate and the abscissa now represent an electron and a hole, respectively.

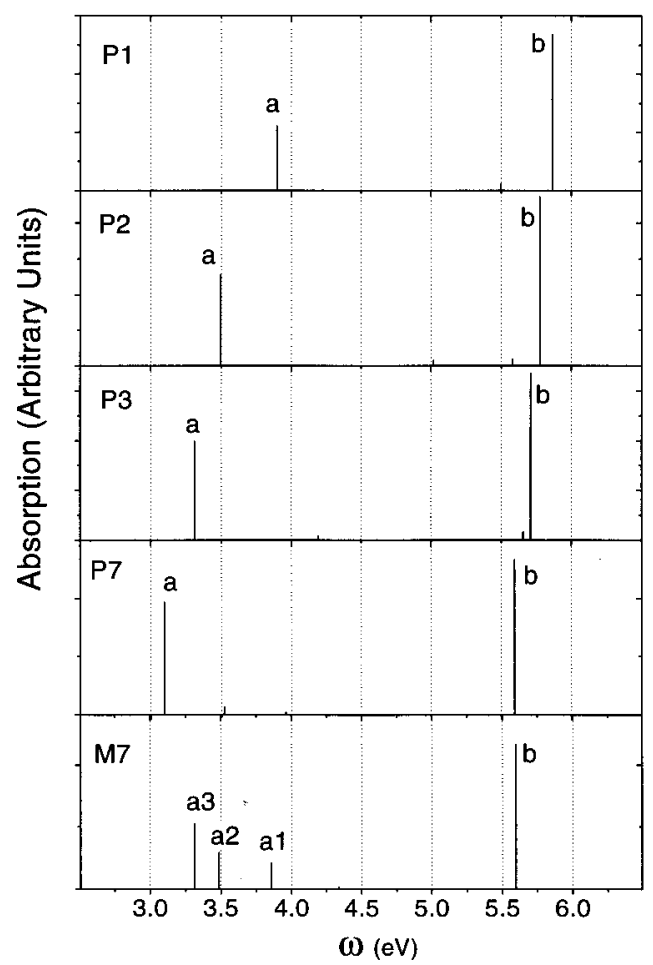

Figure 4. Calculated linear absorption (oscillator strengths $f_{v}$ vs transition frequencies $\Omega_{v}$ ) of the molecules shown in Figure 3. Mode frequencies of P1 $\left(\Omega_{\mathrm{a}}=3.90 \mathrm{eV}, \Omega_{\mathrm{b}}=5.87 \mathrm{eV}\right) ; \mathrm{P} 2\left(\Omega_{\mathrm{a}}=3.49 \mathrm{eV}\right.$, $\left.\Omega_{\mathrm{b}}=5.78 \mathrm{eV}\right) ; \mathrm{P} 3\left(\Omega_{\mathrm{a}}=3.31 \mathrm{eV}, \Omega_{\mathrm{b}}=5.71 \mathrm{eV}\right) ; \mathrm{P} 7\left(\Omega_{\mathrm{a}}=3.11 \mathrm{eV}\right.$ $\left.\Omega_{\mathrm{b}}=5.60 \mathrm{eV}\right) ; \mathrm{M} 7\left(\Omega_{\mathrm{a} 1}=3.86 \mathrm{eV}, \Omega_{\mathrm{a} 2}=3.48 \mathrm{eV}, \Omega_{\mathrm{a} 3}=3.31 \mathrm{eV}\right.$, $\left.\Omega_{\mathrm{b}}=5.60 \mathrm{eV}\right)$.

The right column displays the high-frequency transition $b$ in P-oligomers. This mode is completely localized on a single repeat unit, and the optically induced coherences (off-diagonal elements) only involve the arene atoms. Its localized nature leads to the similar charge and coherence patterns in M7(b) and P7(b) (and in the high-frequency mode in PPV oligomers ${ }^{8}$ ). This explains the similarity of peak $b$ in the absorption spectra of M7 and P7.

The middle column shows the lowest-frequency (band edge) mode a of the para-oligomers. P1(a) centered at the triple bond shows maximum coherences and is delocalized over the entire molecule. Carbons 2, 3, 12, and 13, which are at meta-position, have vanishing electronic coherences with other carbon atoms. This is shown by the "ring" around the plot with small coherences. Analogous patterns can be seen in mode a of longer linear oligomers P2, P3, and P7. The mode saturates with size and is no longer confined by the molecular ends, already in P7. These plots clearly illustrate the two characteristic length scales corresponding to the variation of the density matrix along the "antidiagonal" and the "diagonal" directions, respectively. The former reflects the size of electron-hole pair created upon optical excitation, (i.e., the confinements of their relative motion). The latter shows the delocalization size of the pair's center of mass motion and represents energy migration across the molecule. We shall refer to these as the exciton coherence and localization sizes, respectively. We find that the coherence size (where the coherences decrease to $10 \%$ of their maximum values), is five repeat units, in agreement with that found in ref 8 for PPV oligomers. The boundary meta-atoms $(2,3,8 n+4$, $8 n+5)$ have vanishing coherences in all P-oligomers.

The left column in Figure 6 displays the electronic modes of M7. Mode a3 is localized at the P3 linear segment of M7 and is virtually identical to mode a of the P3 oligomer. Similarly 

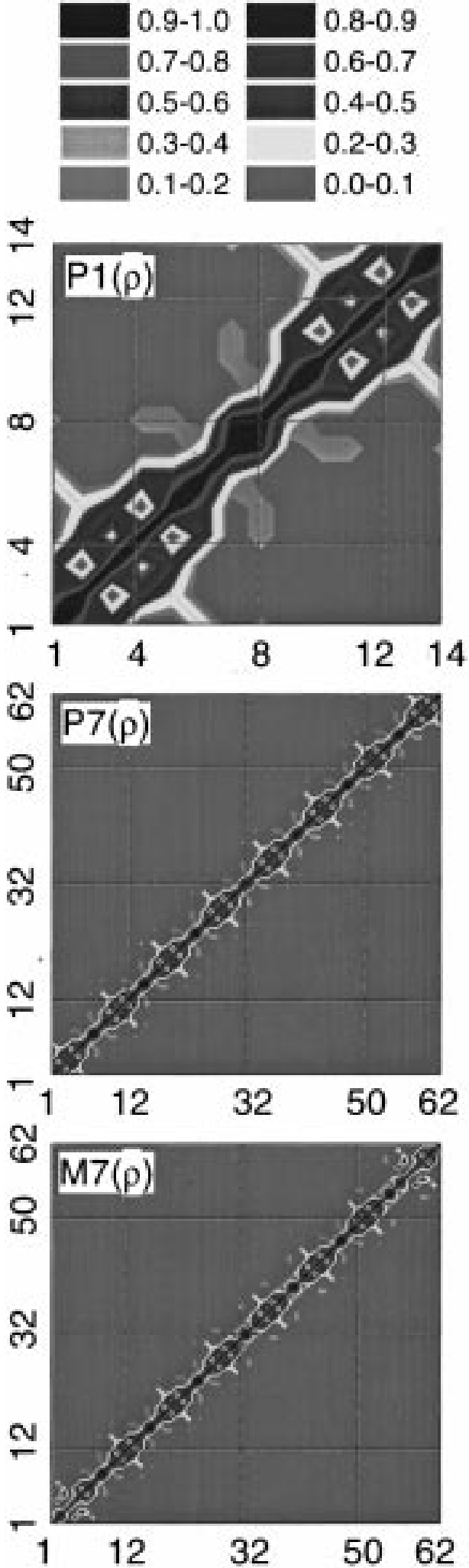

Figure 5. Contour plots of the ground-state density matrixes of oligomers P1, P7, and M7. The axes represent the carbon atoms as labeled in Figure 3. The panels are labeled the molecule (Figure 3) and the electronic mode (Figure 4) (e.g., P1 $(\rho)$ is the ground-state density matrix of molecule $\mathrm{P} 1$ ). The aromatic ring units are shown by solid rectangles. The color code is given in the top panel.

$\mathrm{M} 7(\mathrm{a} 2)$ and $\mathrm{M} 7(\mathrm{a} 1)$ resemble $\mathrm{P} 2$ (a) and P1(a), respectively. Note that $\mathrm{a} 1$ is degenerate because M7 has two identical P1 building units. The complete absence of coherence across metasubstitution shown in this figure is remarkable. The optical excitations are clearly confined to the various segments. Metaconjugation makes a clear barrier for excitonic motion, whereas para-conjugation is transparent to electronic coherences. This difference does not show up in the ground state, which is very similar in both cases (see Figure 5).

It is well-established that meta-substituents are much less effective in changing chemical reaction rates compared with their para-counterparts. ${ }^{22}$ This can be understood using resonant structures which show that charges injected into the system by a nuclophilic or an electrophilic substituent are delocalized only at the ortho- and para-positions. The present study, which establishes the same trend for electron-hole pairs created by light, provides a direct link between spectroscopy and wellestablished rules of thumb for chemical reactivity.

\section{Spectra and Energy Funneling in Dendrimers}

Electronic excitations in molecular aggregates made out of chromophores with nonoverlaping charge distributions may be described as Frenkel excitons. ${ }^{23,24}$ In these systems, electron exchange is negligible and each chromophore has its own electrons. Frenkel excitons are tightly bound electron-hole pairs that hop coherently or incoherently across the aggregate. At first glance this picture does not apply to dendrimers which have a conjugated electronic structure, and their single-electron states (molecular orbitals) are fully delocalized across the entire molecule. However, our analysis shows that while the electronhole pairs of molecules conjugated at the para-position are delocalized, their motions are sharply confined by metaconjugation. Individual molecular orbitals are not particularly useful in the interpretation of spectra since they are dominated by pairs of orbitals. It is the localization of these pairs as shown in the electronic normal modes that controls the nature of optical excitations. An important and very surprising consequence of the present study is that the Frenkel exciton picture is not restricted to chromophores with nonoverlapping charge distributions; It can be safely used as long as the electronic modes are spatially separated and the quasiparticles of the system are localized.

The lack of electronic coherence across meta-substitutions suggests that we can describe the optical excitations of dendrimers by dividing them into weakly interacting chromophores separated by the meta-substitutions. In zero-order we can neglect the interactions among chromophores altogether, and the oligomer's optical spectrum is then simply given by the sum of the spectra of its segments that are connected at the metapositions. The meta-conjugated dendrimer behaves as a collection of its linear para-conjugated segments that interact with light independently.

To calculate the absorption spectra of dendrimers ${ }^{11-13}$ we modeled family A as a collection of P1 chromophores. The spectra will thus only show one low-frequency peak a1 whose intensity will increase with growing molecular size. Equation 1.3 with the empirical line width $\Gamma=0.02 \mathrm{eV}$ is used to calculate the modeled spectra of dendrimers. The experimental and the modeled spectra of D-4 and D-10 members of family A are displayed in Figure 2. The spectra of other generations of this family are very similar. ${ }^{11}$

We have calculated the absorption spectra of the B family by simply adding the spectra of its segments. For example D-58 consist of 3 units of P3, 6 units of P2, and 36 units of P1. We multiplied the corresponding oscillator strengths $f_{\mathrm{P} 3}, f_{\mathrm{P} 2}$, and $f_{\mathrm{P} 1}$ by the number of absorbing units and used eq 1.3 to calculate the spectrum. The resulting calculated and experimental spectra of extended dendrimers B are compared in Figure 2. The plots show that this simple procedure can produce the band edge red- 

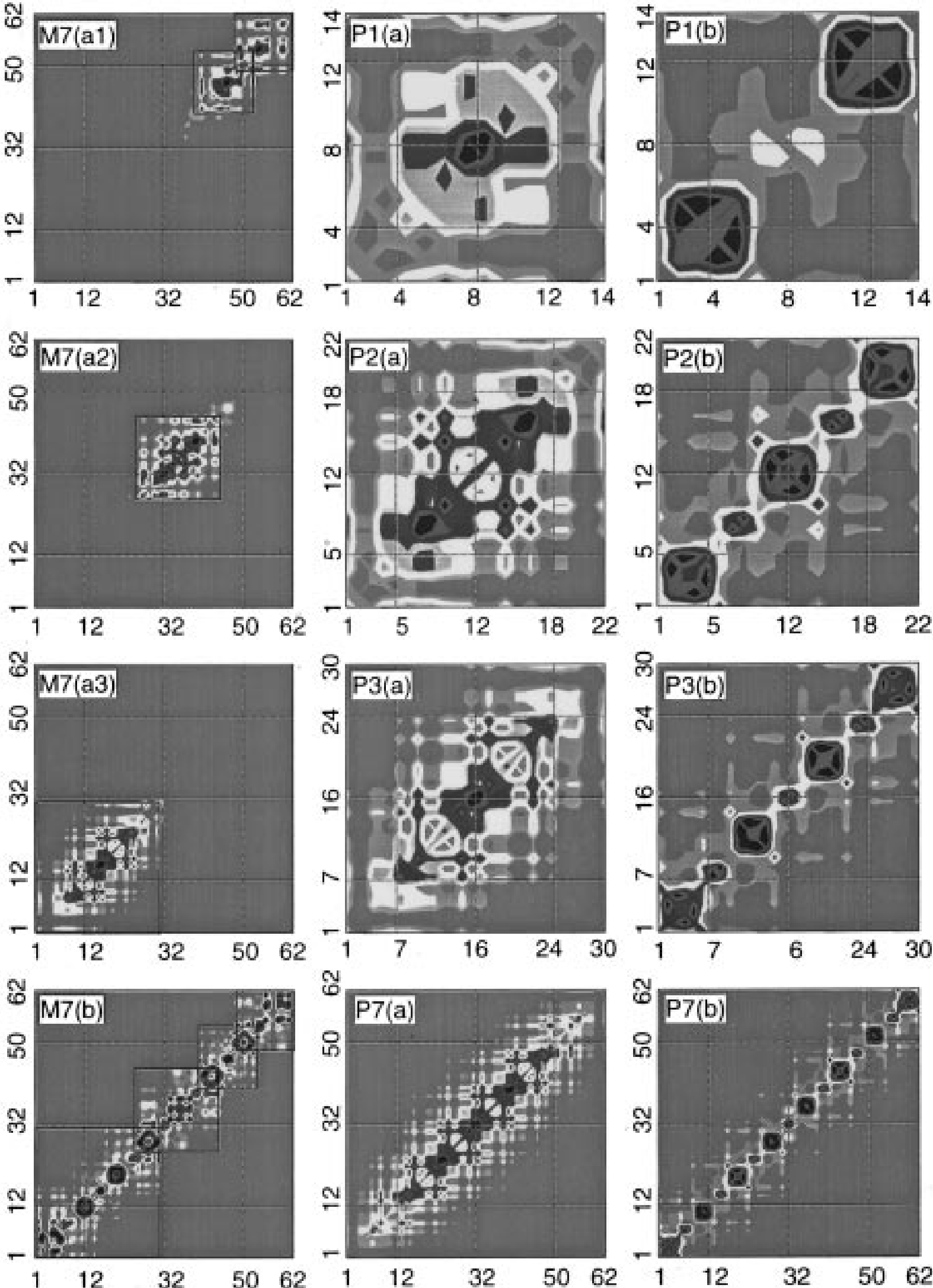

Figure 6. Contour plots of the electronic modes that dominate the absorption spectra of the oligomers shown in Figure 3 . The axes represent the carbon atoms as labeled in Figure 3. The panels indicate the molecule (Figure 3) and the electronic mode (Figure 4). The linear units in M7 are shown by solid rectangles. The color code is given in Figure 5.

shift trend as well as relative peak intensities in these macromolecules, in complete agreement with experiment.

The lack of electronic coherence across the meta-positions implies that electron (and hole) exchange is blocked, in contrast to the para-and ortho-positions, which allow a significant charge delocalization. However, Coulomb interactions between segments do allow the transfer of energy through the migration of electron-hole pairs (excitons). This motion may be either 
coherent or incoherent via the Forster-Dexter mechanism. ${ }^{23,24}$ We estimate the electrostatic interaction between electronic modes on neighboring segments chemically bonded through the meta-positions to be $\sim 500 \mathrm{~cm}^{-1}$. This value is supported by direct calculations of absorption spectra in the compact (family A) dendrimers, which show a weak Davydov-like splitting $\sim 200-600 \mathrm{~cm}^{-1}$ in the band-edge transition. This is a typical value for $J$ aggregates,${ }^{25}$ biological antenna complexes,${ }^{26}$ and molecular crystals. ${ }^{23,24}$ The CEO approach can be readily applied for very large molecules with thousands of atoms. The lack of long-range electronic coherence allows us to truncate the density matrix and only retain off-diagonal elements of closely lying atoms. ${ }^{27}$ This results in most favorable linear $\mathrm{N}$-scaling of computational effort with size, resembling similar developments in ground-state calculations. ${ }^{28}$ The CEO can be extended to account for nuclear motions by incorporating additional nuclear oscillators. The time-dependent density matrix should then allow us to follow the dynamics of coherent intramolecular and intermolecular vibrations, solvent modes, and isomerization and account for vibronic structure and line broadenings. ${ }^{29}$ An important consequence of the present study is the ability to break the electronic excitations of dendrimers into several chromophores, despite the delocalized nature of the underlying electronic states. This provides a useful guideline for designing artificial light-harvesting antennae: by adjusting the lengths of the para-substituted segments in each generation, it should be possible to control the funneling of energy to a desired site. Antennae such as family B have an energy gradient that favors the migration of energy toward the center where a reactive site can be placed.

Acknowledgment. We thank Dr. S. F. Swallen and Prof. R. Kopelman for providing us with the linear absorption data and for most valuable discussions. We also greatly appreciate the enlighting comments of Prof. J. Klafter. S.M. wishes to thank the support of the Guggenheim Fellowship, the Alexander Von Humboldt Award, and the kind hospitality of Prof. Ed Schlag at the Technical University of Munich. The support of the Air Force Office of Scientific Research (Grant No. F4962096-1-0030), and the National Science Foundation through Grants No. CHE-9526125 and No. PHY94-15583, is gratefully acknowledged. The calculations were conducted using the resources of the Cornell Theory Center, which receives major funding from the NSF and New York State.

\section{References and Notes}

(1) Tomalia, D. A.; Naylor, A. M.; Goddard, W. A. Angew. Chem., Int. Ed. Engl. 1990, 29, 138.
(2) Jiang, D.-L.; Aida, T. Nature 1997, 388, 454.

(3) Mukamel, S. Nature 1997, 388, 425.

(4) Fox, M. A.; Jones, W. E.; Watkins, D. M. Chem. Eng. News 1993 , $71,38$.

(5) Bar-Haim, A.; Klafter, J.; Kopelman, R. J. J. Am. Chem. Soc. 1997 $119,6197$.

(6) Junge, D. M.; McGrath, D. V. Chem. Commun. 1997, 857.

(7) Shortreed, M.; Swallen, S. F.; Shi, Z.-Y.; Tan, W.; Xu, Z.; Devadoss, C.; Moore, J. S.; Kopelman, R. J. Phys. Chem. B 1997, 101, 6318.

(8) Mukamel, S.; Tretiak, S.; Wagersreiter, T.; Chernyak, V. Science 1997, 277, 781. Chernyak, V.; Mukamel, S. J. Chem. Phys. 1996, 104, 444.

(9) Tretiak, S.; Chernyak, V.; Mukamel, S. J. Chem. Phys. 1996, 105, 8914. Tretiak, S.; Chernyak, V.; Mukamel, S. J. Am. Chem. Soc. 1997, 119,11408 .

(10) Gust, D. Nature 1997, 386, 21.

(11) Kopelman, R.; Shortreed, M.; Shi, Z.-Y.; Tan, W.; Xu, Z.; Moore, J. S.; Bar-Haim, A.; Klafter, Y. Phys. Rev. Lett. 1997, 78, 1239.

(12) Shortreed, M.; Shi, Z.-Y.; Kopelman, R. Mol. Cryst. Liq. Cryst. 1996, $283,95$.

(13) Devadoss, C.; Bharathi, P.; Moore, J. S. J. Am. Chem. Soc. 1996, $118,9635$.

(14) Zyss, J., Chemla, D. S., Eds. Nonlinear Optical Properties of Organic Molecules and Crystals; Academic Press: Orlando, FL, 1987; Vol. $1, \mathrm{p} 2$.

(15) Brédas, J. L.; Adant, C.; Tackyx, P.; Persoons, A.; Pierce, B. M. Chem. Rev. 1994, 94, 243.

(16) Szabo, A.; Ostlund, N. S. Modern Quantum Chemistry: Introduction to Advanced Electronic Structure Theory; McGraw-Hill: New York, 1989.

(17) Milliken, R. S. J. Chem. Phys. 1955, 23, 1833, 1841, 2338, 2343.

(18) Lowdin, P. O. Phys. Rev. 1955, 97, 1474; Adv. Phys. 1956, 5, 1.

(19) J. A. Pople; Segal, G. A. J. Chem. Phys. 1965, 43, S136. Pople, J. A.; Beveridge, D. L.; Dobosh, P. J. Chem. Phys. 1967, 47, 2026.

(20) Ridley, J.; Zerner, M. C. Theor. Chim. Acta 1973, 32, 111.

(21) Zerner, M. C.; Loew, G. H.; Kirchner, R. F.; Mueller-Westerhoff, U. T. J. Am. Chem. Soc. 1980, 102, 589.

(22) Michl, J.; Bonacic-Koutecky, J. Electronic Aspects of Organic Photochemistry; Willey: New York, 1990. Klessinger, M.; Michl, J. Excited States and Photochemistry of Organic Molecules, VCH: New York, 1995.

(23) Pope, M.; Swenberg, C. E. Electronic Processes in Organic Crystals; Clarendon Press, Oxford University Press: Oxford: New York, 1982.

(24) Silinsh, E. A.; Čápek, V. Organic Molecular Crystals; American Institute of Physics Press: New York, 1994.

(25) van Burgel, M.; Wiersma, D. A.; Duppen, K. J. Chem. Phys. 1995, $102,20$.

(26) Sauer, K.; Cogdell, R. J.; Prince, S. M.; Freer, A. A.; Isaacs, N. W.; Scheer, H. Photochem. Photobiol. 1996, 64, 564.

(27) Dubovsky, O.; Mukamel, S. J. Chem. Phys. 1991, 95, 7828.

(28) Schwegler, E.; Challacombe, M.; Head-Gordon, M. J. Chem. Phys. 1997, 106, 9708. Strain, M. C.; Scuseria, G. E.; Frisch, M. J. Science 1996, 271,5245 .

(29) Zerbi, G.; Galbiati, E.; Gallazzi, M. C.; Castiglioni, C.; Del Zoppo, M.; Schenk, R.; Mölen, K. J. Chem. Phys. 1996, 105, 2509. 